\title{
Transients due to Instabilities Hinder Kardar-Parisi-Zhang Scaling: A Unified Derivation for Surface Growth by Electrochemical and Chemical Vapor Deposition
}

\author{
Rodolfo Cuerno ${ }^{1}$ and Mario Castro ${ }^{2,3}$ \\ ${ }^{1}$ Departamento de Matemáticas \& GISC, Universidad Carlos III de Madrid, Avenida Universidad 30, 28911 Leganés, Spain \\ ${ }^{2}$ Universidad Pontificia de Comillas, 28015 Madrid, Spain \\ ${ }^{3}$ Departamento de Física de Materiales \& GISC, Facultad de Ciencias Físicas, Universidad Complutense de Madrid, \\ 28040 Madrid, Spain
}

\begin{abstract}
We propose a unifie moving boundary problem for surface growth by electrochemical and chemical vapor deposition, which is derived from constitutive equations into which stochastic forces are incorporated. We compute the coefficient in the interface equation of motion as functions of phenomenological parameters. The equation features the Kardar-Parisi-Zhang (KPZ) nonlinearity and instabilities which, depending on surface kinetics, can hinder the asymptotic KPZ scaling. Our results account for the universality and the experimental scarcity of KPZ scaling in the growth processes considered.
\end{abstract}

DOI: 10.1103/PhysRevLett.87.236103

PACS numbers: 68.35.Ct, 64.60.Ht, 81.15.Gh, 81.15.Pq

The dynamics of rough surfaces [1] is a subject of high interest. This is due both to its implications for processes of technological relevance $[2,3]$, and to the interesting instances that it offers of extended systems evolving in the presence of fuctuations [4]. A very successful framework for the study of rough interfaces has been the use of stochastic growth equations for the interface height. Among these, the one proposed by Kardar, Parisi, and Zhang (KPZ) [5] has played a prominent role, since in particular it has enabled connections to be made with other physical problems, such as directed polymers in disordered media or randomly stirred fuids [1]. On the basis of a coarse-grained description of surface growth and symmetry arguments, the KPZ equation was initially expected to describe the dynamics of surfaces growing, e.g., at the expense of a vapor phase, in the absence of conservation laws, and is thus expected to be relevant to such diverse physical growth systems as electrochemical deposition (ECD) [2] or chemical vapor deposition (CVD) [3]. The generality of KPZ scaling would be a consequence of the phenomenon of universality observed for the scaling properties of rough surfaces. However, to date very few experiments have been reported which are compatible with the predictions of the KPZ equation [6-8]. Moreover, mere symmetry arguments do not enable a detailed connection with phenomenological parameters describing specifi experimental systems, while detailed derivations of the KPZ equation were achieved for discrete or continuous theoretical models [9], only indirectly related with experiments. These facts have led to invoking additional effects on the same coarse-grained level, such as specifi noise statistics, nonlocal effects, etc. [1], in order to account for the difference between the observed and the predicted scaling behaviors of rough surfaces. However, a wide range of scaling exponents ensued, there being no theoretical argument that could identify the correct exponents for a specifi growth experiment.
In this Letter we study two of the main techniques employed in experiments on nonconserved surface growth, namely ECD and CVD. These techniques have actually played a preeminent role in the study of pattern formation [10], but only recently have they been shown to provide experimental realizations of rough interfaces in the KPZ universality class [7,8]. The asymptotic behavior is in both systems preceded by exceedingly long unstable transients, extending in, e.g., the experiments in [8] for up to two days deposition time. This complex time behavior is thus far unaccounted for on general grounds by any coarse-grained continuum model. Here we start from the constitutive equations of ECD and CVD, into which we allow for stochastic forces, following a similar treatment to that employed in studies of solidificatio [11], step dynamics [12], or fuid imbibition [13]. We show that ECD and CVD can both be described within a unifie framework, which provides a stronger statement on universality in nonconserved growth phenomena than that restricted to scaling behavior. Moreover, we compute the coefficient appearing in the ensuing stochastic interface equation of motion (IEOM) as functions of the phenomenological parameters characterizing the corresponding physical growth process. The IEOM features the expected KPZ nonlinearity, but also instabilities which can hinder asymptotic KPZ scaling. Specificall, for the case of noninstantaneous growth events at the surface, the IEOM is a stochastic generalization $[12,14]$ of the Kuramoto-Sivashinsky equation [15], for which very long transients due to instabilities are known to occur [16] before scaling behavior can be observed. Thus, there is no need to invoke additional effects at a coarse-grained level in order to account for the difficult in observing KPZ scaling, but rather they are due to the long unstable transients which will quite generically occur. Our approach also accounts for features of discrete growth models [17] and our conclusions are thus expected to apply rather generally for nonconserved growth systems. 
We f rst consider growth by CVD. A successful model of this type of growth was formulated and developed in [18]. A stagnant diffusion layer of inf nite vertical extent is assumed to exist above the substrate upon which an aggregate will grow. Particles of an intermediate species [concentration $c(x, z, t) \equiv c(\mathbf{r}, t)$, where $x$ is the coordinate along the initial one-dimensional substrate and $z$ is the growth direction] diffuse through the stagnant layer. When they meet the surface they react in order to stick to the aggregate, this occurring with an eff ciency measured by a kinetic mass-transfer coeff cient $k_{D}$ [19]. Additional curvature driven effects, such as surface diffusion and evaporation condensation, can infuence the local growth velocity of the aggregate. Moreover, we will take into account local fuctuations in the vapor phase and surface diffusion currents, as well as in the deposition events, in order to account for the experimental relevance of fuctuations for the morphology of surfaces grown by CVD [8]. We thus propose the following stochastic generalization of the deterministic model of CVD [18]:

$$
\begin{gathered}
\frac{\partial c}{\partial t}=D \nabla^{2} c-\nabla \cdot \mathbf{q}, \\
k_{D}\left(c-c_{\mathrm{eq}}^{0}-\Gamma \kappa+\chi\right)=(D \nabla c-\mathbf{q}) \cdot \mathbf{n}, \\
\mathbf{v} \cdot \mathbf{n}=\Omega(D \nabla c-\mathbf{q}) \cdot \mathbf{n}-B \nabla_{\mathrm{s}}^{2} \kappa-\Omega \nabla \cdot \mathbf{p}, \\
c(x, z \rightarrow \infty, t)=c_{a} .
\end{gathered}
$$

In Eq. (1), $D$ is the diffusion constant, and the conserved noise $\mathbf{q}(\mathbf{r}, t)$ represents f uctuations in the concentration associated with diffusion through the stagnant layer. Equations (2) and (3) hold at any point on the aggregate surface $\mathbf{r}=\mathbf{s}$, the symbol $\nabla_{\mathrm{s}}^{2}$ denoting the surface Laplacian operator and $\mathbf{n}$ the local unit normal vector. Equation (2) is a mixed boundary condition which relates the diffusion current arriving at the aggregate from the stagnant layer with the material which actually deposits, via the kinetic coeff cient $k_{D}$. The noise term $\chi$ represents fuctuations in the deposition events [12], $c_{\mathrm{eq}}^{0}$ is the equilibrium concentration for a $\mathrm{f}$ at interface, $\boldsymbol{\kappa}$ is the local mean curvature, and $\Gamma=\gamma c_{\mathrm{eq}}^{0} \Omega /\left(k_{B} T\right)$, with $\gamma$ the surface tensionwhose anisotropy will be neglected, i.e., we will consider an amorphous or polycrystalline aggregate - and $T$ the temperature. In Eq. (3), $\mathbf{v}$ is the local aggregate velocity, $\Omega$ is the atomic volume of the depositing species, and the coeff cient of the surface diffusion current [1] $B=$ $D_{\mathrm{s}} \nu_{\mathrm{s}} \gamma \Omega^{2} / k_{B} T$, with $D_{\mathrm{s}}$ the surface diffusivity and $\nu_{\mathrm{s}}$ the surface concentration of particles. Moreover, $\mathbf{p}$ is a noise term associated with the surface diffusion current. Finally, $c_{a}$ in (4) is a constant value held f xed at the edge of the stagnant layer. Note that the deterministic model of CVD [18] is recovered by neglecting $\mathbf{q}, \mathbf{p}$, and $\chi$ in (1)-(4). We consider zero-mean, uncorrelated, and white noise terms. A local equilibrium hypothesis [11,12] then allows us to determine their variances to be [20]

$$
\begin{aligned}
& \left\langle q^{i}(\mathbf{r}, t) q^{j}\left(\mathbf{r}^{\prime}, t^{\prime}\right)\right\rangle=2 D c(\mathbf{r}, t) \delta_{i j} \delta\left(\mathbf{r}-\mathbf{r}^{\prime}\right) \delta\left(t-t^{\prime}\right) \\
& \left\langle p^{i}(\mathbf{s}, t) p^{j}\left(\mathbf{s}^{\prime}, t^{\prime}\right)\right\rangle=2 D_{\mathrm{s}} \nu_{\mathrm{s}} \delta_{i j} \delta\left(\mathbf{s}-\mathbf{s}^{\prime}\right) \delta\left(t-t^{\prime}\right) \\
& \left\langle\chi(\mathbf{s}, t) \chi\left(\mathbf{s}^{\prime}, t^{\prime}\right)\right\rangle=\left(2 c(\mathbf{s}, t) / k_{D}\right) \delta\left(\mathbf{s}-\mathbf{s}^{\prime}\right) \delta\left(t-t^{\prime}\right)
\end{aligned}
$$

Before studying the interface dynamics predicted by model (1)-(4), let us show that it also describes electrochemical deposition, under a proper interpretation of the f elds and parameters appearing. For simplicity, we assume a rectangular thin cell in which the two electrodes are made of the same metal, the cell being flled with a dilute solution of a salt of this metal. In a growth experiment by ECD [2], an electric $f$ eld is applied driving the motion of cations towards the cathode, whereupon they stick via a reduction reaction, leading to the growth of an aggregate. The constitutive equations, neglecting convection of the electrolyte, [21] describe diffusion of cations (concentration $C$ ) and of anions (concentration $A$ ), together with Poisson's equation for the electric feld across the cell. This highly nontrivial system can be somewhat simplif ed under the electroneutrality condition [22] $z_{a} A=z_{c} C$, where $e z_{c}$ and $-e z_{a}$ are the cationic and anionic charges, implying

$$
\partial_{t} C=D \nabla^{2} C,
$$

where $D=\left(\mu_{c} D_{a}+\mu_{a} D_{c}\right) /\left(\mu_{a}+\mu_{c}\right)$ is the ambipolar diffusion constant, with $\mu_{c, a}$ being the cationic and anionic mobilities. The electric feld conf guration and the anion dynamics are implicit in the def nition of $D$ and in the boundary conditions (BC), which we need to specify. The simplest BC are that the anion $\mathrm{fux}$ be zero both at the anode and at the cathode, where only cations contribute to the aggregate growth. The current density at the cathode surface is then [2]

$$
\mathbf{J} \cdot \mathbf{n}=-\left[z_{c} D_{c} F /\left(1-t_{c}\right)\right] \nabla C \cdot \mathbf{n},
$$

where $t_{c} \equiv \mu_{c} /\left(\mu_{a}+\mu_{c}\right)$ and $F$ is Faraday's constant. Moreover, charge transport at the cathode is an activated process, whose balance is described by the Butler-Volmer equation $[2,23]$

$$
J=J_{0}\left[e^{(1-\beta) \eta z_{c} F / R T}-e^{-\left(\beta \eta+\eta_{s}\right) z_{c} F / R T} C / C_{a}\right],
$$

where $J_{0}$ is the exchange current density in equilibrium, $\beta$ is a coeff cient between 0 and 1 describing the asymmetry of the energy barrier related to the cation reduction reaction, $C_{a}$ is the initial cation concentration, $R$ is the gas constant, and $\eta$ is the overpotential, from which a surface curvature contribution $\eta_{s}$ has been singled out. By def ning the concentration feld $c \equiv D_{c} C /\left[D\left(1-t_{c}\right)\right]\left(c_{a}\right.$ and $c_{\mathrm{eq}}^{0}$ are def ned accordingly) and performing an expansion of Eq. (10) for a small value of $\eta_{s}=\Omega^{\mathrm{ECD}} \gamma^{\mathrm{ECD}} R \kappa /$ $\left(z_{c} F k_{B}\right)$ [2], Eqs. (1)-(4) provide the stochastic generalization of the ECD model (8)-(10) incorporating surface tension and surface diffusion effects at the aggregate surface. Note that Eqs. (9) and (10) together amount to a 
mixed BC [24] on $c$ of the type of Eq. (2), with a kinetic coeff cient $k_{D}^{\mathrm{ECD}} \equiv J_{0} e^{-\beta z_{c} F \eta / R T} D\left(1-t_{c}\right) /\left(z_{c} F C_{a} D_{c}\right)$. For instance, the $\eta \rightarrow-\infty$ limit of completely eff cient reduction at the cathode leads to an absorbing boundary condition there of the type $c=c_{\mathrm{eq}}^{0}+\Gamma \kappa$.

We are now in a position to study the generic model (1)-(4) and draw conclusions for the two diverse growth systems considered. We follow a similar approach to that in [11-13], and references therein. Namely, we f rst note that in the zero noise limit Eqs. (1)-(4) support a fat solution $c(\mathbf{r}, t)=c(z, t)$, moving at a constant velocity $V=$ $k_{D}\left[\Omega\left(c_{a}-c_{\mathrm{eq}}^{0}\right)-1\right]$. By the use of the diffusion Green function, we project the moving boundary problem onto the aggregate surface. Finally, we perform a perturbation expansion and a long wavelength analysis in order to derive a stochastic differential equation for a local deviation $\zeta(x, t)$ (in the frame moving with velocity $V$ ) from the $\mathrm{f}$ at interface solution. Details will be reported elsewhere [20]. The results are conveniently classif ed by the value of the kinetic coeff cient $k_{D}$ [25].

Instantaneous surface kinetics $\left(k_{D} \rightarrow \infty\right)$. - Denoting by $\zeta_{k}(t)$ the $k$ th Fourier mode of $\zeta(x, t)$, in the case of inf nitely fast reaction kinetics at the interface (absorbing boundary condition) the IEOM reads

$$
\partial_{t} \zeta_{k}(t)=\omega(k) \zeta_{k}(t)+\frac{V}{2} \mathcal{F}_{k}\left[(\nabla \zeta)^{2}\right]+\beta_{k}^{\mathrm{absorb}}(t) .
$$

Here $\mathcal{F}_{k}[f(x)]$ denotes the $k$ th Fourier mode of $f(x)$, the nonlinear term in (11) having the expected KPZ form, and $\beta_{k}^{\text {absorb }}(t)$ is an additive noise term whose correlations depend on the dispersion relation $\omega(k)$ [26]. Note that, in principle, the system (1)-(4) has multiplicative noise. However, similarly to [12], to lowest nonlinear order in $\zeta(x, t)$ the IEOM features only additive noise terms. For Eq. (11) the dispersion relation reads $\omega(k)=$ $V|k|\left(1-d_{0} l_{D} k^{2}\right)\left[1-d_{0} / l_{D}+\left(d_{0}^{2} / 4-B / D\right) k^{2}\right]^{1 / 2}+$ $D\left(d_{0}^{2} / 2-B / D\right) k^{4}-3 d_{0} D k^{2} / l_{D}$, where we have def ned a capillarity length $d_{0}=\Gamma \Omega$ and a diffusion length $l_{D}=D / V$. As we see, due to the shape of $\omega(k)$, the IEOM (11) is nonlocal in space. This is a refection of the diffusional instabilities present in the system [10]. For instance, in the absence of surface diffusion currents $(B=0)$, and as long as $d_{0} \ll l_{D}$, the dispersion relation of Eq. (11) is of the Mullins-Sekerka type [10], $\omega(k) \simeq$ $V|k|\left(1-d_{0} l_{D} k^{2}\right)$. However, the IEOM does have additive noise and a KPZ nonlinear term with coeff cient $V / 2$, both facts as expected on general grounds [1]. Although the behavior of Eq. (11) at large scales is not completely known, both a scaling argument and preliminary numerical simulations indicate that KPZ scaling is not asymptotic under these growth conditions [27].

Noninstantaneous surface kinetics $\left(k_{D}<\infty\right)$. - For the case of noninstantaneous deposition events at the surface, the dispersion relation $\omega(k)$ turns out to depend only on even powers of $k$, which allows to express the IEOM directly in conf guration space, featuring only local terms [we omit the $\mathbf{r}$ and $t$ dependencies of $\zeta(\mathbf{r}, t)$ ]:

$$
\partial_{t} \zeta=-a_{2} \nabla^{2} \zeta-a_{4} \nabla^{4} \zeta+\frac{V}{2}(\nabla \zeta)^{2}+\beta^{\operatorname{mixed}}(\mathbf{r}, t),
$$

where $a_{2}=k_{D} l_{D} \Delta, a_{4}=k_{D} l_{D}^{2} \Delta d_{0} /\left[1-\left(d_{0} / l_{D}\right)^{1 / 2}\right]+$ $B\left(1+k_{D} / V\right)$, and $\Delta=1-d_{0} / l_{D}$. Again, $\beta^{\operatorname{mixed}}(\mathbf{r}, t)$ is an additive noise with $\omega(k)$-dependent correlations [26]. As in Eq. (11), the coeff cient of the KPZ nonlinearity is $V / 2$. However, while (11) always has a band of linearly unstable modes, this happens in Eq. (12) only if $\Delta>0$, i.e., when surface tension is unable to counteract the diffusional instabilities $\left(d_{0}<l_{D}\right)$. In this unstable case, (12) is the stochastic Kuramoto-Sivashinsky (KS) equation, already encountered in other interface dynamics contexts, such as step dynamics [12], or surface erosion by ion-beam sputtering [14]. In the stochastic KS system, there is a linearly most unstable mode $k_{m}=\left(2 l_{D} d_{0}\right)^{-1 / 2}$ (assuming $B=0)$, whose onset time is $\omega_{m}^{-1}=2 /\left(k_{D} l_{D} k_{m}^{2}\right)$. For asymptotic times, KPZ scaling is obtained, but only after an exceedingly long transient [16].

From the above analysis of Eqs. (11) and (12), KPZ scaling should be expected only for slow surface kinetics, and will, nevertheless, be affected by early time instabilities, unless the capillary length is larger than the typical diffusion length in the system. KPZ scaling corresponds to the conformal growth mode, which is most interesting for applications of CVD-grown flms and can indeed be achieved under industrial conditions by tuning the relative values of $k_{D}, l_{D}$, and $d_{0}[3,8,18]$. In the case of ECD, we can further verify the predictions from model (1)-(4) via Eqs. (11), (12) with both experimental [7,28] and Monte Carlo [17] studies. For instance, in the ECD experiment in [28] the diffusion length $l_{D} \approx 2 \mathrm{~cm}$ is the largest length scale in the system, and a Mullins-Sekerka dispersion relation is reported; consequently, after a stable transient the diffusional instabilities that occur completely override any scaling behavior at long times. As a difference, in the experiments of [7] the growing aggregate undergoes an unstable transient, beyond which its surface stabilizes into the KPZ stationary state. This behavior is qualitatively compatible with that of the noisy KS equation: from the experimental value of the branch spacing one has $k_{m} \simeq 1.3 \times 10^{3} \mathrm{~cm}^{-1}$. Using [7] $D \simeq$ $10^{-5} \mathrm{~cm}^{2} \mathrm{~s}^{-1}$ and $V \simeq 2 \times 10^{-4} \mathrm{~cm} \mathrm{~s}^{-1}$, one obtains a typical value $d_{0}=1 /\left(2 l_{D} k_{m}^{2}\right) \simeq 5 \times 10^{-6} \mathrm{~cm}$, hence indeed $d_{0} \ll l_{D} \simeq 0.05 \mathrm{~cm}$. Moreover, the instability occurs after $1 / \omega_{m} \simeq 0.3 \times 10^{3} \mathrm{~s}$, which allows one to estimate $k_{D} \simeq 8 \times 10^{-8} \mathrm{~cm} \mathrm{~s}^{-1} \ll V$, hence conditions are in the slow kinetics regime.

Equations (1)-(4) also apply to the dynamics of discrete growth models such as the multiparticle biased diffusion limited aggregation (MBDLA) model [17], which is a generalization of the DLA model $[1,10]$ to the case of a f nite concentration of random walkers performing biased 
random walks (bias parameter $p$ ), which stick to the growing aggregate with a f nite sticking probability $s$. MBDLA reproduces ECD experiments for one-dimensional substrates quantitatively [17], and ECD experiments for two-dimensional substrates qualitatively [29]. Thus, the sticking probability $s$ plays the role of a noise reduction parameter, in the sense that for small $s$ values the system reaches faster its KPZ asymptotic scaling behavior; this role is played in our continuum model by the kinetic mass transfer coeff cient $k_{D}$ [19]. Furthermore, the bias $p$ is proportional to the aggregate velocity $V$. As the characteristic branch spacing is, according to (12), approximately equal to $\left(l_{D} d_{0}\right)^{-1 / 2}$, the continuum model predicts a ramif edto-compact transition as $p$ increases, such as is observed in MBDLA [17]. Moreover, since the coeff cient of the KPZ term also increases, the corresponding scaling is expected to occur earlier as $V$ (or $p$ ) increases, again as is observed in the discrete model.

In summary, our study of CVD and ECD leads us to expect diffusional instabilities to generically hinder KPZ scaling in nonconserved growth experiments. Our continuum approach from constitutive equations allows us to perform detailed comparison with phenomenological parameters in experiments, and also provides a physical interpretation for features of discrete models of kinetic roughening such as noise-reduction parameters [1], e.g., of the type of the sticking parameter in MBDLA [17]. More detailed predictions from model (1)-(4) would benef $t$ from a computationally more eff cient formulation. Work along these lines is currently under way [20].

We are pleased to acknowledge discussions with and comments by J. Buceta, F. Domínguez-Adame, A. Hernández-Machado, E. Moro, M. A. Rodríguez, M. A. Rubio, A. Sánchez, and L. Vázquez. This work has been supported by DGES (Spain) Grant No. BFM2000-0006.

[1] A.-L. Barabási and H. E. Stanley, Fractal Concepts in Surface Growth (Cambridge University Press, Cambridge, 1995); J. Krug, Adv. Phys. 46, 139 (1997).

[2] J. O'M. Bockris and A. K. N. Reddy, Modern Electrochemistry (Plenum/Rosetta, New York, 1970).

[3] K. F. Jensen and W. Kern, in Thin Film Processes II, edited by J. L. Vossen and W. Kern (Academic, Boston, 1991).

[4] J. García-Ojalvo and J. M. Sancho, Noise in Spatially Extended Systems (Springer, New York, 1999).

[5] M. Kardar, G. Parisi, and Y.-C. Zhang, Phys. Rev. Lett. 56, 889 (1986).

[6] R. Paniago et al., Phys. Rev. B 56, 13442 (1997); J. Maunuksela et al., Phys. Rev. Lett. 79, 1515 (1997).

[7] P. Schilardi et al., Phys. Rev. B 59, 4638 (1999).

[8] F. Ojeda et al., Phys. Rev. Lett. 84, 3125 (2000).

[9] See, e.g., B. Grossmann, H. Guo, and M. Grant, Phys. Rev. A 43, 1727 (1991); L. Bertini and G. Giacomin, Commun.
Math. Phys. 183, 571 (1997).

[10] Y. Saito, Statistical Physics of Crystal Growth (World Scientif c, Singapore, 1996).

[11] A. Karma, Phys. Rev. Lett. 70, 3439 (1993); Phys. Rev. E 48, 3441 (1993).

[12] A. Karma and C. Misbah, Phys. Rev. Lett. 71, 3810 (1993); O. Pierre-Louis and C. Misbah, ibid. 76, 4761 (1996); Phys. Rev. B 58, 2259 (1998); 58, 2276 (1998).

[13] M. Dubé et al., Phys. Rev. Lett. 83, 1628 (1999); Eur. J. Phys. B 15, 701 (2000); A. Hernández-Machado et al., Europhys. Lett. 55, 194 (2001).

[14] R. Cuerno and A.-L. Barabási, Phys. Rev. Lett. 74, 4746 (1995).

[15] Y. Kuramoto and T. Tsuzuki, Prog. Theor. Phys. 55, 356 (1976); G. I. Sivashinsky, Acta Astronaut. 6, 569 (1979).

[16] K. Sneppen et al., Phys. Rev. A 46, R7351 (1992); R. Cuerno et al., Phys. Rev. Lett. 75, 4464 (1995); S. Park et al., ibid. 83, 3486 (1999).

[17] A. Sánchez, M. J. Bernal, and J. M. Riveiro, Phys. Rev. E 50, R2427 (1994); M. Castro et al., ibid. 57, R2491 (1998); 62, 161 (2000).

[18] C. H. J. Van den Brekel and A. K. Jansen, J. Cryst. Growth 43, 364 (1977); B. J. Palmer and R. G. Gordon, Thin Solid Films 158, 313 (1988); G. S. Bales, A.C. Redf eld, and A. Zangwill, Phys. Rev. Lett. 62, 776 (1989).

[19] The coeff cient $k_{D}$ has dimensions of velocity and is related [18] by kinetic theory with the sticking probability $\tilde{s}$ of particles diffusing near a partially absorbing boundary as $k_{D} \propto D L_{\mathrm{mfp}}^{1} \tilde{s} /(2 \quad \tilde{s})$, with $L_{\mathrm{mfp}}$ the mean free path for the diffusing particle and $D$ the diffusion constant.

[20] M. Castro and R. Cuerno (to be published).

[21] J.-N. Chazalviel, Phys. Rev. A 42, 7355 (1990).

[22] Electroneutrality can be assumed at distances farther away from the cathode than a typical diffusion length; see J.-N. Chazalviel and V. Fleury, Phys. Rev. E 54, 4480 (1996); C. Lèger, J. Elezgaray, and F. Argoul, ibid. 58, 7700 (1998).

[23] D. Kashchiev and A. Milchev, Thin Solid Films 28, 189 (1975).

[24] S. C. Hill and J. I. D. Alexander, Phys. Rev. E 56, 4317 (1997).

[25] The relevance of surface kinetics to the stability properties of the interface is known from work on, e.g., directed solidif cation, M. L. Frankel, Phys. Lett. A 128, 57 (1988).

[26] Noise correlations in Eqs. (11) and (12) depend on the corresponding $\omega(k)$ as $\left\langle\beta_{k}(t) \beta_{k^{\prime}}\left(t^{\prime}\right)\right\rangle=(2 \pi){ }^{1} \Omega^{2} \delta\left(k+k^{\prime}\right) \times$

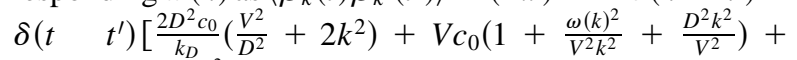
$\left.2 D_{s} \nu_{\mathrm{s}} k^{2} \frac{\left(V+k_{D}\right)^{2}}{k_{D}^{2}}\right]$, with conserved and nonconserved terms.

[27] M. Castro, Ph.D. thesis, Universidad Complutense de Madrid 2001 (unpublished).

[28] J. M. Pastor and M. A. Rubio, Phys. Rev. Lett. 76, 1848 (1996).

[29] With respect to the existence of an intermediate unstable transient with anomalous scaling, and KPZ asymptotic scaling, see S. Huo and W. Schwarzacher, Phys. Rev. Lett. 86, 256 (2001); M. Castro, R. Cuerno, and A. Sánchez (to be published). 